\title{
Polarization Dependence of Second Order Dielectric Constants in Copolymers of Vinylidene Fluoride and Trifluoroethylene
}

\author{
Susumu IKeda, Masayuki Kutani, Takashi Fukaya, \\ and Hiraku Kominami \\ Department of Polymer Materials Engineering, Faculty of Engineering, \\ Yamagata University, Jonan 4-3-16, Yonezawa 992, Japan
}

(Received June 30, 1990)

\begin{abstract}
A second order dielectric constant which represents nonlinear properties of material depends remarkably on the remanent polarization of the material. This paper describes the polarization dependence of second order dielectric constants in ferroelectric copolymers of vinylidene fluoride and trifluoroethylene. The remanent polarization of samples is controlled by means of an electrically or thermally stimulating procedure, both of which depolarize partially the fully polarized sample. The second order dielectric constants of the two sorts of samples made by these two methods show different types of dependence on the remanent polarization. This is interpreted by the phenomenological theory which predicts the thermodynamical order of a ferroelectric phase transition.

KEY WORDS Ferroelectric Polymer / 2nd Order Dielectric Constant / Remanent Polarization / Ferroelectric Phase Transition / Phenomenological Theory /
\end{abstract}

Some fluoro-polymers exhibit ferroelectricity which is defined by the existence of spontaneous polarization and its reversal by application of an electric field. ${ }^{1-3}$ These ferroelectric polymers lose the ferroelectric property at the ferroelectric phase transition temperature (Curie transition temperature). ${ }^{\mathbf{4} 5}$ Recently, many studies of ferroelectric polymers have been focused on the phase transition in copolymers of vinylidene fluoride (VDF) and trifluoroethylene (TrFE). Their transition behavior changes with composition; the Curie transition occurs in the copolymers including more than $50 \mathrm{~mol} \% \mathrm{VDF}$ and the phase transition temperature is increased with increasing mole ratio of the VDF. ${ }^{4}$ Furthermore, copolymers with more than $60 \mathrm{~mol} \%$ VDF exhibit a thermal hysteresis, defined as the difference between the transition temperature in the heating process and that in the cooling one. Thermal hysteresis increases with increasing VDF fraction in the copolymer up to about $80 \mathrm{~mol} \%$. Copolymers with more than $80 \mathrm{~mol} \%$ VDF do not exhibit explicitly the phase transitions because they are covered by melting transitions. From these results, it is presumed that the Curie transition of these copolymers with the VDF fraction above $60 \mathrm{~mol} \%$ is of the first order thermodynamically.

The authors reported the nonlinear dielectric property of the copolymer of VDF and TrFE. $^{6-8}$ The nonlinear dielectric response includes information about polarization reversal occurring by an electric field stimulus above the coercive electric field $E_{\mathrm{c}}$. Furthermore, nonlinear dielectric constants related to nonlinearity in fluctuations of dipoles can be obtained from nonlinear response to an applied electric field below $E_{\mathrm{c}}$.

The $n$-th order dielectric constant $\varepsilon_{n}$ is defined as a coefficient of $E^{n}$ when an electric 
displacement $D$ is expanded by a series of an electric field $E$.

$$
D=\varepsilon_{1} E+\varepsilon_{2} E^{2}+\cdots+\varepsilon_{n} E^{n}+
$$

We measured $\varepsilon_{2}$ and $\varepsilon_{3}$ as functions of temperature and considered them phenomenologically in terms of the free energy expanded as a polynomial of the polarization $P .{ }^{7}$ The results suggest that the temperature dependence of $\varepsilon_{2}$ gives the Curie transition temperature $T_{\mathrm{c}}$ as a vanishing point of $\varepsilon_{2}$ and a change in the sign of $\varepsilon_{3}$ at $T_{\mathrm{c}}$ reflects the thermodynamical order of the phase transition. $^{7}$

In this paper, we describe experimental results about the remanent polarization dependence of $\varepsilon_{2}$ at room temperature and considerations on the coefficient of $P^{n}$ in the free energy which determines the thermodynamical character of the system.

\section{EXPERIMENTAL}

We prepared two sorts of samples differing in the polarization state. The first series of samples with various values of the remanent polarization was prepared by decreasing the electric field to zero from arbitrary intermediate points of a $D-E$ hysteresis curve after the sample had undergone several cycles of the hysteresis (Figure 1). The samples in this case are abbreviated as sample $\mathrm{A}$, whose remanent polarization is denoted as $P_{\mathrm{r} 1}$. The second series of samples was prepared by heating the well-polarized sample to arbitrary temperatures near the Curie temperature and quenching it to room temperature. This sample is named sample B whose remanent polarization is abbreviated as $P_{\mathrm{r} 2}$. (Figure 2) Although $P_{\mathrm{r} 1}$ of the sample $\mathrm{A}$ was determined by an intersection of the hysteresis curve and the vertical axis in Figure $1, P_{\mathrm{r} 2}$ of the sample B was reexamined by integration of the depolarization current in the heating process of the sample up to $T_{\mathrm{c}}$ after its $\varepsilon_{2}$ had been measured. The polarizations of these two types of samples

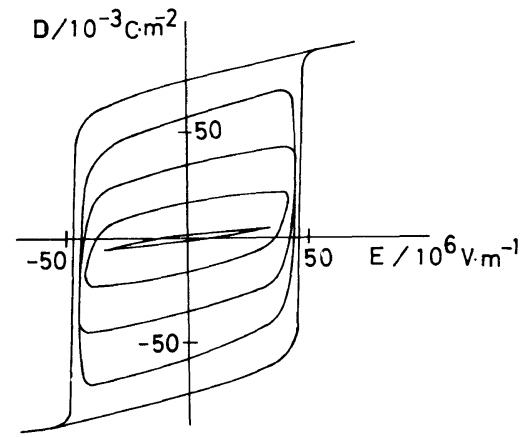

Figure 1. $D-E$ hysteresis in the VDF/TrFE (65/35) copolymer. The inner four hysteresis curves were obtained by reducing the amplitude of the applied field. The remanent polarization $P_{\mathrm{r} 1}$ of the sample A was determined at the intercepts on the ordinate in these curves.

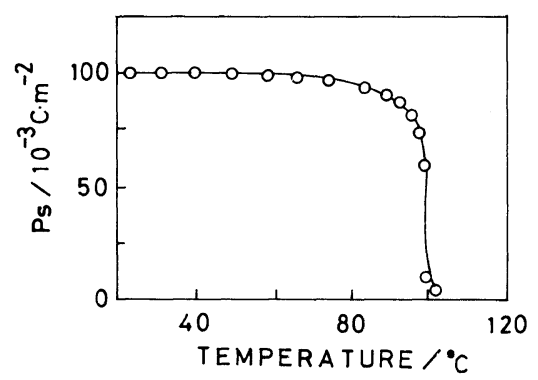

Figure 2. Temperature dependence of $P_{\mathrm{s}}$ for $(65 / 35)$ copolymer. The open circles stand for $P_{\mathrm{s}}$ estimated by integrating TSC curve. Sample B was prepared by quenching from arbitrary temperatures in this heating process to room temperature.

are in different states even if the samples have the same value for the component perpendicular to the film surface of the remanent polarization.

An experimental setup to obtain $\varepsilon_{2}$ was described in a previous paper. ${ }^{7}$ Powders of VDF-TrFE copolymers whose VDF fraction were 52,65 , and $73 \mathrm{~mol} \%$ were supplied by Daikin Kogyo Co. Test films cast from their DMA solutions were annealed at $140^{\circ} \mathrm{C}$ for $2 \mathrm{~h}$ to stabilize their structures and degrees of crystallinity. Gold electrodes with rectangular shape of $5 \mathrm{~mm} \times 8 \mathrm{~mm}$ were evapolated on both surfaces of samples. 


\section{RESULTS}

Figure 3 illustrates the $P_{\mathrm{r} 1}$ dependence of the real part of $\varepsilon_{2}$ in the copolymers $(52 / 48)$, $(65 / 35)$, and $(73 / 27)$. This figure is similar to Figure 8 in ref 7 except for the points the sample films for $(65 / 35)$ and $(73 / 27)$ were cast from their solutions as well as that for the $(52 / 48)$

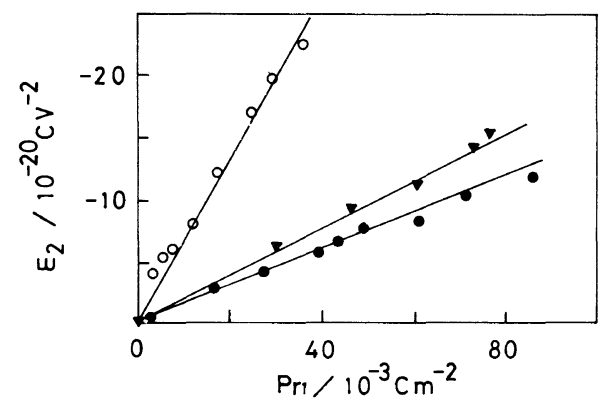

Figure 3. $\varepsilon_{2}-P_{\mathrm{r} 1}$ relations of $\mathrm{VDF} / \mathrm{TrFE}$ copolymers. $\bigcirc$, $(52 / 48) ; \bigcirc,(65 / 35) ; \nabla,(73 / 27)$.

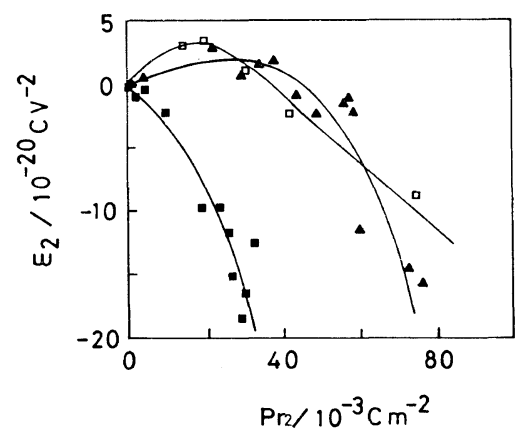

Figure 4. $\varepsilon_{2}-P_{\mathrm{r} 2}$ relations of $\mathrm{VDF} / \mathrm{TrFE}$ copolymers $(52 / 48) ; \square,(65 / 35) ; \boldsymbol{\Delta},(73 / 27)$.

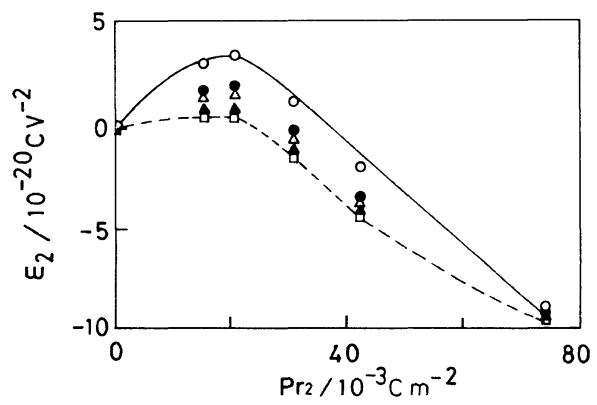

Figure 5. Temporal change in the $\varepsilon_{2}-P_{\mathrm{r} 2}$ relation of $(65 / 35)$ copolymer. Times elapsed from quenching of the samples: $\bigcirc, 300 \mathrm{~s} ; \odot, 600 \mathrm{~s} ; \triangle, 900 \mathrm{~s} ; \Delta, 1800 \mathrm{~s} ; \square, 3600 \mathrm{~s}$. and the slope of the line for the $(73 / 27)$ is slightly larger than that for the $(65 / 35)$. This figure shows that $\varepsilon_{2}$ is proportional to $P_{\mathrm{r} 1}$ and the slope of the line for the $(52 / 48)$ is much larger than those for $(65 / 35)$ and $(73 / 27)$.

In Figure $4, \varepsilon_{2}-P_{\mathrm{r} 2}$ relations for the copolymers $(52 / 48),(65 / 35)$, and $(73 / 27)$ are illustrated. We find from this figure that the relation between $\varepsilon_{2}$ and $P_{\mathrm{r} 2}$ for the sample $B$ is not linear but apparently cubic.

Figure 5 illustrates changes in the $\varepsilon_{2}-P_{\mathrm{r} 2}$ relation with lapse of time after the sample has been quenched.

\section{DISCUSSION}

We prepared two sorts of samples which were different in the state of remanent polarization. The $\varepsilon_{2}-P_{\mathrm{r} 1}$ relation observed for the sample $\mathrm{A}$ was linear. On the other hand, the $\varepsilon_{2}-P_{\mathrm{r} 2}$ relation observed for the sample $B$ gave an apparent cubic function. These results may be explained in terms of the phenomenological free energy expanded as a polynomial of $P$.

The elastic Gibbs energy for ferroelectric materials is

$$
\begin{aligned}
G-G_{0}= & (1 / 2) \beta\left(T-T_{0}\right) P^{2} \\
& +(1 / 4) \gamma P^{4}+(1 / 6) \delta P^{6}
\end{aligned}
$$

where $G_{0}, \beta, \gamma$, and $\delta$ are constants. From this equation we can obtain $\varepsilon_{2}$.

$$
\varepsilon_{2}=-\left(3 \gamma+10 \delta P_{\mathrm{s}}{ }^{2}\right) P_{\mathrm{s}}\left(\varepsilon_{1}-\varepsilon_{0}\right)^{3}
$$

where $\varepsilon_{0}$ is the permittivity of free space and $P_{\mathrm{s}}$ is the spontaneous polarization of a single domain. In eq $2, \beta$ and $\delta$ are positive and $\gamma$ is positive or negative according as the phase transition is of the second order or the first order, respectively.

The polarization reversal process by application of the external electric field above $E_{\mathrm{c}}$ has been interpreted from the viewpoint of nucleation and growth. ${ }^{9-11}$ Accordingly, the sample situated on the halfway of the polarization reversal process is composed roughly of two sorts of domains with the spontane- 
ous polarization $P_{\mathrm{s}}$ or $-P_{\mathrm{s}}$ which has the direction parallel or antiparallel to the electric field, respectively. The domain with $P_{\mathrm{s}}$ is separated by the domain wall from the domain with $-P_{\mathrm{s}}$ and each domain is relatively independent of each other.

The linear relation between $\varepsilon_{2}$ and $P_{\mathrm{r} 1}$ is derived from the above consideration. The remanent polarization is written as

$$
P_{\mathrm{r} 1}=v_{+} P_{\mathrm{s}}-v_{-} P_{\mathrm{s}}
$$

where $v_{+}\left(v_{-}\right)$stands for the volume fraction of the domain with $P_{\mathrm{s}}\left(-P_{\mathrm{s}}\right)$. Then we have

$$
\begin{aligned}
\varepsilon_{2} & =(1 / 2)\left(v_{+}-v_{-}\right) \mathrm{d}^{2} P /\left.\mathrm{d} E^{2}\right|_{E=0} \\
& =-\left(3 \gamma+10 \delta P_{\mathrm{s}}{ }^{2}\right) P_{\mathrm{r} 1}\left(\varepsilon_{1}-\varepsilon_{0}\right)^{3}
\end{aligned}
$$

$\varepsilon_{2}$ is proportional to $P_{\mathrm{r} 1}$. This derivation is based on the assumption that the dielectric response of the system is the sum of the respective dielectric response of each domain which possesses $P_{\mathrm{s}}$ or $-P_{\mathrm{s}}$.

On the other hand, the dipole orientation of the thermally treated samples with $P_{\mathrm{r} 2}$ is distributed randomly corresponding to the annealing temperatures because thermal excitation breaks the polarization partly and homogeneously. The orientation distribution of dipoles is supposed to be frozen by quenching.

If the system is composed of elements distributed randomly in the dipole orientation, we cannot treat it in fashion such that the system consists of two independent domains. In this case, the decrease in polarization is not due to cancellation among the domains with different polarizations but due to freezing of dipole fluctuation in the domain. Since the decrease in polarization due to the dipole fluctuation occurs uniformly in the sample, the system may be composed of the single domain. $\varepsilon_{2}$ of the system is written as eq 3 where $P_{\mathrm{s}}$ is rewritten by $P_{\mathrm{r} 2}$.

$$
\varepsilon_{2}=-\left(3 \gamma+10 \delta P_{\mathrm{r} 2}^{2}\right) P_{\mathrm{r} 2}\left(\varepsilon_{1}-\varepsilon_{0}\right)^{3}
$$

This equation does not give a linear relation but a cubic function. This explains the $\varepsilon_{2}-P_{\mathrm{r} 2}$ relation produced by sample $B$.

In the $\varepsilon_{2}-P_{\mathrm{r} 2}$ relations of $(65 / 35)$ and $(73 / 27)$ in Figure 4, the initial positive rise suggests a negative value of $\gamma$, resulting in the first order phase transition. On the other hand, the $\varepsilon_{2}-P_{\mathrm{r} 2}$ relation of the $(52 / 48)$ copolymer shows that $\gamma$ of this copolymer is positive, suggesting the second order transition. This conclusion for the sign of $\gamma$ explains well also the experimental facts shown in Figure 3 where the slope of the $\varepsilon_{2}-P_{\mathrm{r} 1}$ relation in $52 / 48$ copolymer is larger than those of $65 / 35$ and $73 / 27$ copolymers.

The temporal change shown in Figure 5 teaches us that the crystal structure and distribution of the polarization orientation approach the equilibrium domain structure, where the dielectric response of each domain is independent of the other. This shows that the quenching of the sample near $T_{\mathrm{c}}$ results in freezing the state of the copolymer near $T_{\mathrm{c}}$.

We conclude from the $\varepsilon_{2}-P_{\mathrm{r} 2}$ relations that the Curie transition of $65 / 35$ and $73 / 27$ copolymers is of the first order and that of $52 / 48$ copolymer is of the second order. These results agree well with the results obtained by measurement of a temperature dependence of $\varepsilon_{3}$.

Since the second order dielectric constant is the third rank tensor, the value vanishes in the system with a symmetrical center. The spontaneous polarization breaks the symmetry of the domain. The remanent polarization, formed as a vector sum of the spontaneous polarization of the domains, breaks the symmetry of the sample. The second order dielectric constant is thus a powerful means to obtain information about the remanent polarization of a material.

Acknowledgment. The authors are greatly indebted to Daikin Kogyo Co. for providing the samples of VDF-TrFE copolymers. 


\section{REFERENCES}

1. M. Jimbo, S. Kobayashi, K. Horino, S. Ikeda, and Y. Wada, J. Polym. Sci., Polym. Phys. Ed., 22, 2139 (1984).

2. S. Ikeda, M. Jimbo, S. Kobayashi, and Y. Wada, $J$. Polym. Sci., Phys. Ed., 23, 1781 (1985).

3. T. Furukawa, M. Date, and E. Fukada, J. Appl. Phys., 51, 1135 (1980).

4. K. Tashiro, K. Takano, M. Kobayashi, Y. Chatani, and H. Tadokoro, Ferroelectrics, 57, 297 (1984).
5. K. Koga and H. Ohigashi, J. Appl. Phys., 59, 2142 (1986).

6. S. Ikeda, H. Suzuki, K. Koyama, and Y. Wada, Polym. J., 19, 681 (1987).

7. S. Ikeda, H. Kominami, K. Koyama, and Y. Wada, J. Appl. Phys., 62, 3339 (1987).

8. S. Ikeda, H. Kominami, K. Koyama, and Y. Wada, Ferroelectrics, 76, 421 (1987).

9. M. Date and T. Furukawa, Ferroelectrics, 57, 37 (1984).

10. Y. Takase and A. Odajima, Jpn. J. Appl. Phys., 22, L318 (1983). 